\title{
Transplantation of Transduced Chondrocytes Protects Articular Cartilage from Interleukin 1-Induced Extracellular Matrix Degradation
}

\author{
Vijaykumar M. Baragi, * Richard R. Renkiewicz, * Hollis Jordan, “ Jeffrey Bonadio, ${ }^{\ddagger}$ John W. Hartman, ${ }^{\S}$ \\ and Blake J. Roessler $\$$ \\ *Parke-Davis Pharmaceutical Research, Division of Warner-Lambert Company, Ann Arbor, Michigan 48105; Department of \\ Pathology, University of Michigan Medical Center, Ann Arbor, Michigan 48109-0650; and ${ }^{\S}$ Department of Internal Medicine, Division \\ of Rheumatology, University of Michigan Medical Center, Ann Arbor, Michigan 48109-0680
}

\begin{abstract}
Gene therapy used in the context of delivering a therapeutic gene (s) to chondrocytes offers a new approach for treating chondrocyte-mediated cartilage degradation associated with various human arthropathies including osteoarthritis. In this study, gene delivery to human osteoarthritis chondrocytes in monolayer culture was demonstrated using two adenoviral vectors (Ad.CMVlacZ and Ad.RSVntlacZ) carrying the Escherichia coli $\beta$-galactosidase marker gene, and a third vector (Ad.RSV hIL-1ra) containing the cDNA for human interleukin-1 receptor antagonist. At an moi of $10^{3}$ plaque-forming units/chondrocyte, $>90 \%$ of the infected cells stained positive for $E$. coli $\beta$-galactosidase activity, indicating a high efficiency of transduction. Genetically modified chondrocytes were then transplanted onto the articular surface of osteoarthritic cartilage organ cultures with and without the underlying subchondral bone. Both in situ staining of the cartilage organ cultures for $E$. coli $\beta$-galactosidase activity and examination by scanning electron microscopy indicated that the transplanted chondrocytes adhered and integrated into the articular surface and continued to express transgenic protein. Chondrocytes transduced with Ad.RSV hIL-1ra and seeded onto the surface of osteoarthritic cartilage secreted high levels of biologically active IL-1 receptor antagonist. The Ad.RSV hIL-1ra-treated cartilage samples were resistant to IL1-induced proteoglycan degradation over $10 \mathrm{~d}$ of sustained organ culture. These data demonstrate that transplantation of transduced chondrocytes onto the articular surface protects cartilage from IL-1-induced extracellular matrix degradation. (J. Clin. Invest. 1995. 96:2454-2460.) Key words: arthritis - gene therapy $\bullet$ interleukin-1 receptor antagonist $\bullet$ adenovirus
\end{abstract}

\section{Introduction}

Chondrocyte-mediated degradation of articular cartilage has been suggested as a major factor in the pathogenesis of various

Address correspondence to Blake J. Roessler, University of Michigan Medical Center, 5520 MSRB I, Box 0680, Ann Arbor, MI 48109-0680. Phone: 313-747-3413; FAX: 313-763-4151. Address reprint requests to Vijaykumar M. Bargi, Department of Immunopathology, Parke-Davis/ Warner-Lambert Company, 2800 Plymouth Road, Ann Arbor, MI 48105.

Received for publication 22 May 1995 and accepted in revised form 21 June 1995.

J. Clin. Invest.

(C) The American Society for Clinical Investigation, Inc.

0021-9738/95/11/2454/07 \$2.00

Volume 96, November 1995, 2454-2460 human arthropathies including osteoarthritis (OA) ${ }^{1}(1-6)$. Therapeutic strategies aimed at developing biological chondroprotective agents are faced with the challenge of targeting these proteins to the immediate microenvironment of articular chondrocytes. These problems are exacerbated by the fact that cartilage is an avascular and largely nonreplicative tissue. Given the limitations of traditional methods of biological drug delivery, gene therapy offers a new approach for the possible treatment of human arthritis. Both ex vivo and in vivo techniques using viral and nonviral vectors have been used to deliver the Escherichia coli $\beta$-galactosidase (lac $Z$ ), neomycin resistance genes, and the IL-1 receptor antagonist (IL-1ra) cDNA to synoviocytes (7-12). However, these same gene delivery techniques used to target synoviocytes have been unsuccessful in delivering genes to articular chondrocytes. Although delivery of expression vectors encoding soluble proteins to synoviocytes has been shown to be effective in inhibiting proinflammatory cytokine effects, the direct effect of these maneuvers on chondrocytemediated cartilage degradation remains to be determined ( 10 , 11). These results once again emphasize the need for the development of models for direct gene transfer to articular chondrocytes.

Recently, it has been demonstrated that human chondrocytes can be expanded in tissue culture and used for autologous transplantation onto articular cartilage. When these cells are applied to full thickness cartilage defects and sealed over with a periosteal flap, this treatment can lead over time to the growth of tissue within the defect that resembles normal articular cartilage (13). This treatment has only been applied to more or less solitary lesions in an otherwise normal joint, and it has yet to be seen whether chondrocyte transplantation alone would be sufficient to heal the defects associated with OA cartilage. Nevertheless, the success of autologous chondrocyte transplantation suggests it may be used in combination with ex vivo gene transfer to enhance the therapeutic potential of these cells.

The degradation of cartilage extracellular matrix (ECM) observed in OA is a complex process that at some level can be defined as the end result of catabolic processes outstripping anabolic processes $(5,6)$. IL-1, a cytokine present in OA joint fluid $(14,15)$ has direct (chondrocyte-mediated) and indirect (synoviocyte-mediated) catabolic effects on articular cartilage $(15,16)$. These effects include generation and maintenance of synovial inflammation (17), as well as up-regulation of matrix metalloproteinase (18) and PG expression (19).

We hypothesized that IL-1-mediated catabolic effects on articular chondrocytes are amenable to inhibition via transgenic overexpression of IL-1 antagonists by chondrocytes. Further, we

1. Abbreviations used in this paper: ECM, extracellular matrix; IL-1ra, IL-1 receptor antagonist; OA, osteoarthritis. 
chose to examine the interleukin-1 receptor antagonist protein as a prototypic IL-1 inhibitor. IL-1ra is a naturally occurring protein that is considered to be a potentially therapeutic biological agent $(20,21)$. IL-1ra is a soluble protein that can bind to types I and type II IL-1 receptors but is unable to mediate signal transduction through these receptors (22). There have been a number of in vitro and in vivo studies demonstrating the antiinflammatory effects of $\mathrm{IL}-1 \mathrm{ra}$, and an extensive review of this literature has been published (23).

We have previously reported the construction and functional characterization of a recombinant human adenovirus that can mediate the high level expression of IL-1ra (10). In the present study, we used this adenovirus to mediate gene transfer to human OA chondrocytes in vitro. The transduced cells were then used for transplantation onto the surface of human OA cartilage maintained as a sustained organ culture. The results demonstrate that adenovirally transduced chondrocytes can adhere to and remain viable on the surface of hyaline cartilage and that chondrocytes transduced with the IL-1ra gene can protect OA cartilage from IL-1-induced matrix degradation in this model of ex vivo intraarticular gene therapy.

\section{Methods}

Cartilage organ and chondrocyte cultures. The articular cartilage specimens used in this study were obtained from patients with OA undergoing elective total knee or total hip arthroplasties. The ages of the patients ranged from 50 to $70 \mathrm{yr}$. Tissue samples were obtained after informed consent and performed under a protocol approved by the University of Michigan Institutional Review Board. To establish cartilage organ cultures, uniform cartilage slices measuring $\sim 11 \times 11 \mathrm{~mm}$ were dissected from the underlying bone using a No. 20 scalpel blade and placed in isotonic saline. The cartilage slices were washed in Gey's balanced salt solution (GIBCO BRL, Gaithersburg, MD) and placed (one sample per well) in 24-well flat-bottom tissue culture plates (Falcon Labware, Becton Dickinson, Oxnard, CA). The cartilage organ cultures were maintained in complete Ham's F-12 (GIBCO BRL; supplemented with $10 \%$ FCS, $6.5 \mathrm{mg} / \mathrm{ml}$ Hepes, $58.5 \mu \mathrm{g} / \mathrm{ml}$ glutamine, $200 \mu \mathrm{g} / \mathrm{ml} \mathrm{MgSO}_{4}$, $100 \mathrm{U} / \mathrm{ml}$ penicillin $\mathrm{G}$ sodium, $100 \mu \mathrm{g} / \mathrm{ml}$ streptomycin sulfate, and $0.25 \mu \mathrm{g} / \mathrm{ml}$ amphotericin B ) at $37^{\circ} \mathrm{C}$ in a humidified atmosphere of $95 \%$ air and $5 \% \mathrm{CO}_{2}$.

The chondrocytes needed for the study were isolated directly from the OA cartilage after separation from the underlying bone. The cartilage slices were minced finely and then digested sequentially with $0.2 \%$ testicular hyaluronidase $\left(5 \mathrm{~min}\right.$ at $\left.37^{\circ} \mathrm{C}\right), 0.2 \%$ trypsin $\left(30 \mathrm{~min}\right.$ at $\left.37^{\circ} \mathrm{C}\right)$, and $0.2 \%$ collagenase $\left(1-2 \mathrm{~h}\right.$ at $\left.37^{\circ} \mathrm{C}\right)$. The resulting solutions were strained through sterile nylon mesh and centrifuged at $1,200 \mathrm{~g}$ for 10 min. The cell pellets were washed twice with Ham's F-12 medium, resuspended, pooled, and centrifuged at $1,000 \mathrm{~g}$ for $10 \mathrm{~min}$. The resulting pellet was suspended in complete F-12 medium (described above). This cell suspension was used to establish cell cultures in $25-\mathrm{cm}^{2}$ flasks (Falcon Labware) at a density of 1-2 $11^{6}$ cells $/$ flask. The cells were maintained at $37^{\circ} \mathrm{C}$ in a humidified atmosphere of $95 \%$ air and $5 \% \mathrm{CO}_{2}$. The chondrocytes used in this study were maintained as monolayer cultures for no more than two passages, to maintain the differentiated chondrocyte phenotype.

Recombinant adenoviral vectors. The adenoviral vectors used in this study are based on a human adenoviral (serotype 5) genomic backbone deleted of sequences spanning E1A and E1B and a portion of E3 region. This impairs the ability of this virus to replicate or transform nonpermissive cells (24). Transgene transcription was driven by the early enhancer/promoter of the cytomegalovirus (CMV) in the vector Ad.CMV lacZ and the Rous sarcoma virus (RSV) long-terminal repeat in the vectors Ad.RSV ntlacZ and Ad.RSV hIL-1 ra. An SV40 polyadenylation sequence was cloned downstream from each of these reporters ( 9 ,
10). The vector Ad.RSV ntlac $Z$ contains a nuclear targeting (designated as $n t$ ) epitope linked to the $5^{\prime}$ end of the $l a c Z$ gene, and as a result, the cells transduced with this gene can be identified by the presence of blue nuclei after reaction with the chromogenic substrate 5-bromo-4chloro-3-indolyl- $\beta$-D-galactoside (X-gal).

Transduction of chondrocytes. Primary human chondrocyte cultures were trypsinized and seeded in six-well tissue culture plates at a density of $0.5-1.0 \times 10^{6}$ cells/well. After the cells were $70 \%$ confluent, they were washed two times with serum-free DME, infected with Ad.CMV lacZ, Ad.RSV ntlacZ, or Ad.RSVIL-1ra (viral titers $=10^{10}$ plaque-forming units/ml; diluted 1:100 in serum free DME) at an approximate moi of $10^{3}$ plaque-forming units/cell, for $2 \mathrm{~h}$ at $37^{\circ} \mathrm{C}$. Cells were then washed two times in fresh DME, containing $10 \%$ FCS, 100 $\mathrm{U} / \mathrm{ml}$ penicillin $\mathrm{G}$ sodium, $100 \mu \mathrm{g} / \mathrm{ml}$ streptomycin sulfate, and 0.25 $\mu \mathrm{g} / \mathrm{ml}$ amphotericin B, and the cells were allowed to grow for $24 \mathrm{~h}$ at $37^{\circ} \mathrm{C}$. Mock-infected cells were maintained in parallel. $24 \mathrm{~h}$ after plating, cells were fixed, reacted with X-gal solution, rinsed, and photographed.

Chondrocyte transplantation. Transduced allogeneic chondrocytes were trypsinized, suspended in 1-3 ml of complete Ham's F-12 medium, and added slowly onto the articular surface of cartilage organ cultures or intact cartilage (with the underlying subchondral bone) selected for transplantation experiments. To achieve optimal conditions for adherence of cells to the articular surface, cartilage pieces were pretreated with $10-20 \%$ serum, $0.5 \%$ gelatin, or $3.5 \mu \mathrm{g} \mathrm{Cel}-\mathrm{Tak}^{\circledR}(\mathrm{Col}-$ laborative Biomedical Products, Bedford, MA) $/ \mathrm{cm}^{2}$ surface area. In most of the experiments, complete F-12 (containing 10\% serum) used for maintaining the cartilage organ cultures was sufficient for adherence of cells to cartilage. $24 \mathrm{~h}$ after infection with adenoviral vectors, chondrocytes were trypsinized and added to cartilage organ cultures. Approximately $0.5-1.0 \times 10^{6}$ cells were added to each cartilage piece, and the cultures incubated for $48 \mathrm{~h}$ in complete $\mathrm{F}-12$ medium at $37^{\circ} \mathrm{C}$. In some of the experiments, to prevent cells from settling onto the plastic, the bottom surface of the tissue culture plates were siliconized and cartilage slices placed into these wells before the addition of chondrocytes.

In situ staining for $\beta$-galactosidase transgene expression. Chondrocytes and cartilage organ cultures were washed in PBS and fixed in $2 \%$ paraformaldehyde in $100 \mathrm{mM}$ Pipes, $\mathrm{pH} \mathrm{6.9,} \mathrm{for} 30 \mathrm{~min}$. Samples were washed for $30 \mathrm{~min}$ in PBS/2 $\mathrm{mM} \mathrm{MgCl}$ and reacted in $5 \mathrm{mM} \mathrm{K} \mathrm{K}_{3} \mathrm{Fe}$ $(\mathrm{CN})_{6}, 5 \mathrm{mM} \mathrm{K} \mathrm{Fe}_{4}(\mathrm{CN})_{6}, 2 \mathrm{mM} \mathrm{MgCl}{ }_{2} 0.01 \%$ sodium deoxycholate, $0.02 \% \mathrm{NP}-40$, and $1 \mathrm{mg} / \mathrm{ml} \mathrm{X}$-gal in PBS for $4 \mathrm{~h}$ at $37^{\circ} \mathrm{C}(25)$. Chondrocytes were then washed three times with PBS, postfixed for $2 \mathrm{~h}$ in $2 \%$ paraformaldehyde $/ 1 \%$ glutaraldehyde, counterstained with neutral red, and photographed using light microscopy. At various times (1-8 d) after transplantation, cartilage slices were fixed and reacted with $\mathrm{X}$-gal solution. Parallel samples were trypsinized and the recovered cells plated onto plastic dishes.

Immunohistochemistry. Cells were fixed in $2 \%$ paraformaldehyde, $0.1 \mathrm{M}$ Pipes, $\mathrm{pH} 6.9 / 2 \mathrm{mM} \mathrm{MgCl}_{2}$ then incubated in X-gal solution for $2 \mathrm{~h}$. Cells were washed twice in PBS then processed for immunohistochemistry. Endogenous peroxidase activity was quenched by incubation with $3 \%$ hydrogen peroxide in $90 \%$ methanol for $10 \mathrm{~min}$. Samples were rinsed in PBS then incubated with PBS/10\% FCS containing monoclonal murine anti-human type I collagen (SP1D8; Chemicon International Inc., Temecula, CA) or type II collagen (B1330; Chemicon International Inc.) for $1 \mathrm{~h}$. Cells were washed three times then incubated with goat antimurine IgG conjugated to biotin for $10 \mathrm{~min}$ (Histostain; Zymed Laboratories, Inc., So. San Francisco, CA). Samples were washed then incubated with streptavidin-horseradish peroxidase conjugate for $10 \mathrm{~min}$ followed by reaction with aminoethyl carbazole. Cells were lightly counterstained with hematoxylin and examined using light microscopy for the presence of red precipitate indicating the expression of type I or type II collagen and blue nuclei indicating the coexpression of transgenic lac $Z$.

Scanning electron microscopy. Cartilage slices used for transplantation experiments were examined by scanning electron microscopy to identify integration of transduced cells into the articular surface. Cartilage disks analyzed by scanning electron microscopy were trimmed by sharp dissection to a width of $1 \mathrm{~mm}$ and fixed in $4 \%$ paraformaldehyde 
in PBS followed by $1 \%$ glutaraldehyde in $0.5 \mathrm{M}$ cacodylate buffer. Disks were dehydrated in graded alcohols, desiccated under vacuum, and coated with elemental gold, then imaged using a scanning electron microscope (ISI DS-130, Topcon, Milpitas, CA).

Effects of exogenous IL-1 administration on transgene expression and ECM degradation in vitro. $24 \mathrm{~h}$ after infection, the chondrocytes were trypsinized, washed, and plated onto human OA cartilage organ cultures, and the cultures were incubated in complete F-12 medium at $37^{\circ} \mathrm{C} .48 \mathrm{~h}$ after transplantation of chondrocytes, cartilage organ cultures were rinsed with $1 \mathrm{ml} \mathrm{F-12}$ nutrient mixture (GIBCO BRL) (without serum) to remove residual FCS. Each treatment group was done in triplicate. The controls received $1 \mathrm{ml} \mathrm{F}-12$ medium $+10 \mu \mathrm{l}$ PBS with $0.1 \%$ BSA and IL-1 treatment group received $1 \mathrm{ml} \mathrm{F}-12$ medium +10 $\mu \mathrm{l} \mathrm{IL-1} \alpha(500 \mathrm{U})$. Cartilage organ cultures were incubated for $10 \mathrm{~d}$. Every 48 or $72 \mathrm{~h}$ conditioned medium was replaced with fresh medium and IL-1 or PBS/BSA was added. All media changes were collected. At the end of the experiment, the cartilage pieces were subjected to papain digestion: cartilage pieces were digested in 24-well plates by adding $50 \mu \mathrm{l}$ papain in $1.0 \mathrm{ml}$ cysteine buffer $(0.1 \mathrm{M}$ phosphate buffer, $\mathrm{pH} 7.0$, containing $0.1 \mathrm{M}$ L-cysteine $\mathrm{HCl}$ and $0.05 \mathrm{M}$ EDTA) and incubated at $56^{\circ} \mathrm{C}$ until the cartilage pieces were dissolved completely. The effect of IL-1 on proteoglycan degradation was determined by analyzing the cartilage digests and media samples collected for chondroitin sulfate content, using the modified 1,9-dimethylmethylene blue assay (26). The conditioned media were also analyzed by ELISA (R\& D Systems Inc., Minneapolis, MN) and the total amount of human IL1 ra (endogenous and transgenic) was calculated. Statistical analysis of the data was performed using one way ANOVA followed by StudentNewman-Keul's method of pairwise multiple comparisons.

\section{Results}

Transduction of human chondrocytes. To determine if human chondrocytes in culture are susceptible to infection and transduction by adenoviral vectors, lac $Z$ was used as a marker gene. Two recombinant human serotype 5 ( sub360 derivative) adenovirus vectors, Ad.RSV ntlacZ and Ad.CMV lacZ were used to deliver the marker gene. The vector Ad.RSV ntlac $Z$ contained a nuclear targeting epitope linked to the lac $Z$ gene, thus transduced cells could be identified by the presence of blue nuclei after reaction with the chromogenic substrate X-gal. Greater than $90 \%$ of the infected cells exhibited blue nuclei, indicating transgenic expression of the lac $Z$ gene mediated by adenoviral gene transfer (Fig. $1 A$ ). Similar results were obtained with cells infected with the Ad.CMV lacZ vector, the cells exhibited blue cytoplasmic staining (data not shown). Control cells (mock infected) exhibited no blue staining, indicating that the expression of $\beta$-galactosidase activity was specific for the transgene and did not represent endogenous enzyme activity (data not shown). No abnormalities in cell shape or evidence of cell death were observed in the successfully transduced cells.

Immunohistochemical staining of the transduced chondrocytes indicated that most of the lacZ-positive cells $(\sim 70 \%)$ coexpressed type II collagen, characteristic of a differentiated chondrocyte phenotype (Fig. $1 B$ ). However, as expected for chondrocytes maintained in monolayer culture, a percentage of cells $(\sim 40 \%)$ also coexpressed type I collagen (Fig. 1 C). These experiments demonstrate that human chondrocytes in monolayer culture are readily infected with recombinant adenovirus and that viral episomes efficiently mediate transduction (gene transfer) and transgenic protein expression in vitro. Furthermore, transduced cells retain the ability to manufacture ECM proteins that are characteristic of a differentiated chondrocyte phenotype.
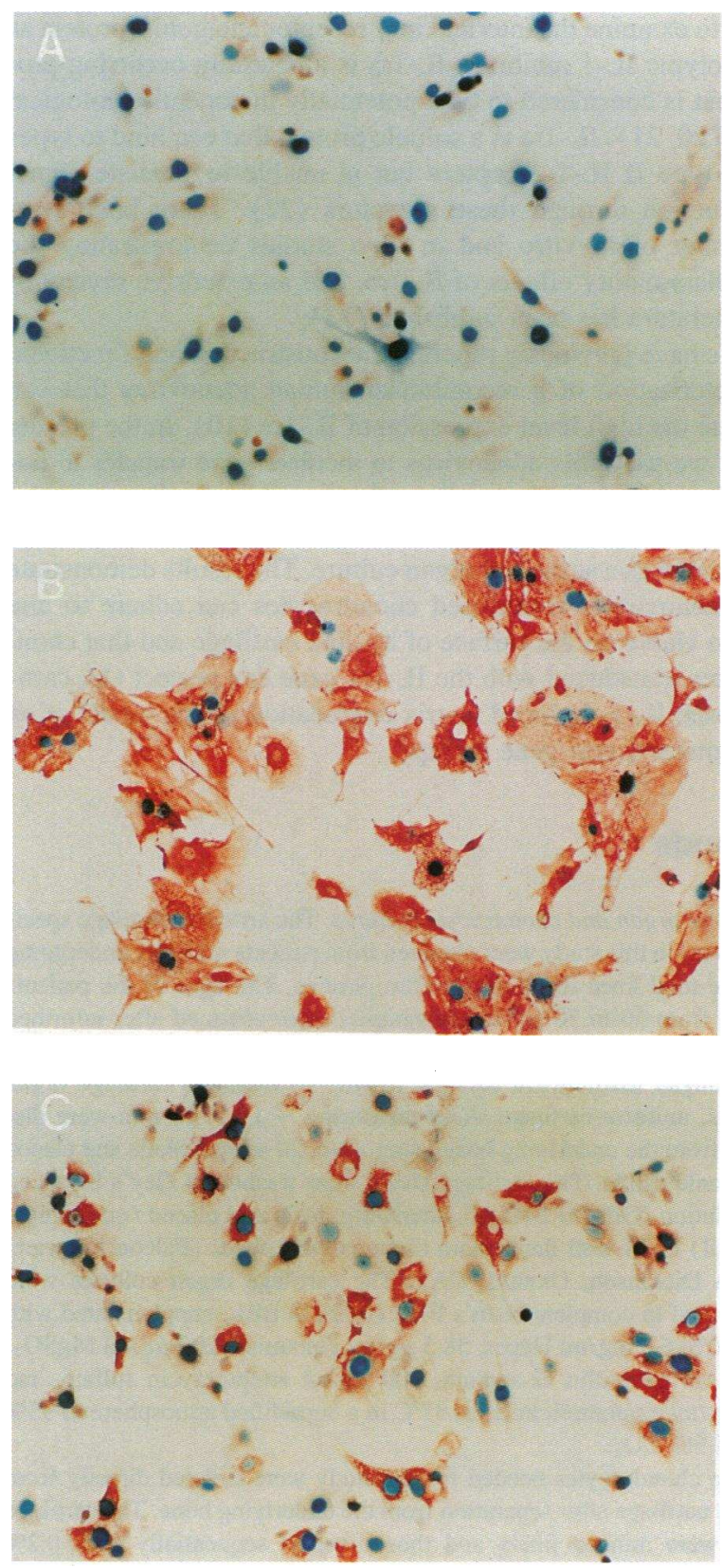

Figure 1. Expression of transgenic lacZ by human chondrocytes infected with Ad.RSV $n$ tlac $Z$ in vitro. Human chondrocytes in monolayer culture were infected with Ad.RSV ntlacZ at an moi of $10^{3}$ plaque forming units/cell. $24 \mathrm{~h}$ after infection, chondrocytes were fixed, then reacted with X-gal, and counterstained with hematoxylin (as described in Methods). Examination by inverted light microscopy (TMS; Nikon Inc.) revealed chondroyctes expressing transgenic $n$ tlac $Z$ as indicated by blue-staining nuclei $(A)$. Duplicate aliquots of $n$ tlac $Z$-transduced chondroyctes were also immunohistochemically stained using mAbs against human type II $(B)$ or type I $(C)$ collagen and a goat anti-marine IgG biotin-streptavidin peroxidase-aminoethyl carbazole indicator system. Transduced chondrocytes were able to coexpress $n$ tlac $Z$ (blue precipitate) in addition to both type II $(B)$ and/or type $\mathrm{I}(C)$ collagen (red precipitate). $\times 200$.

Transplantation of transduced chondrocytes. Transplantation of chondrocytes transduced with the $l a c Z$ and IL-1ra genes were undertaken as a means to overexpress transgenic proteins 

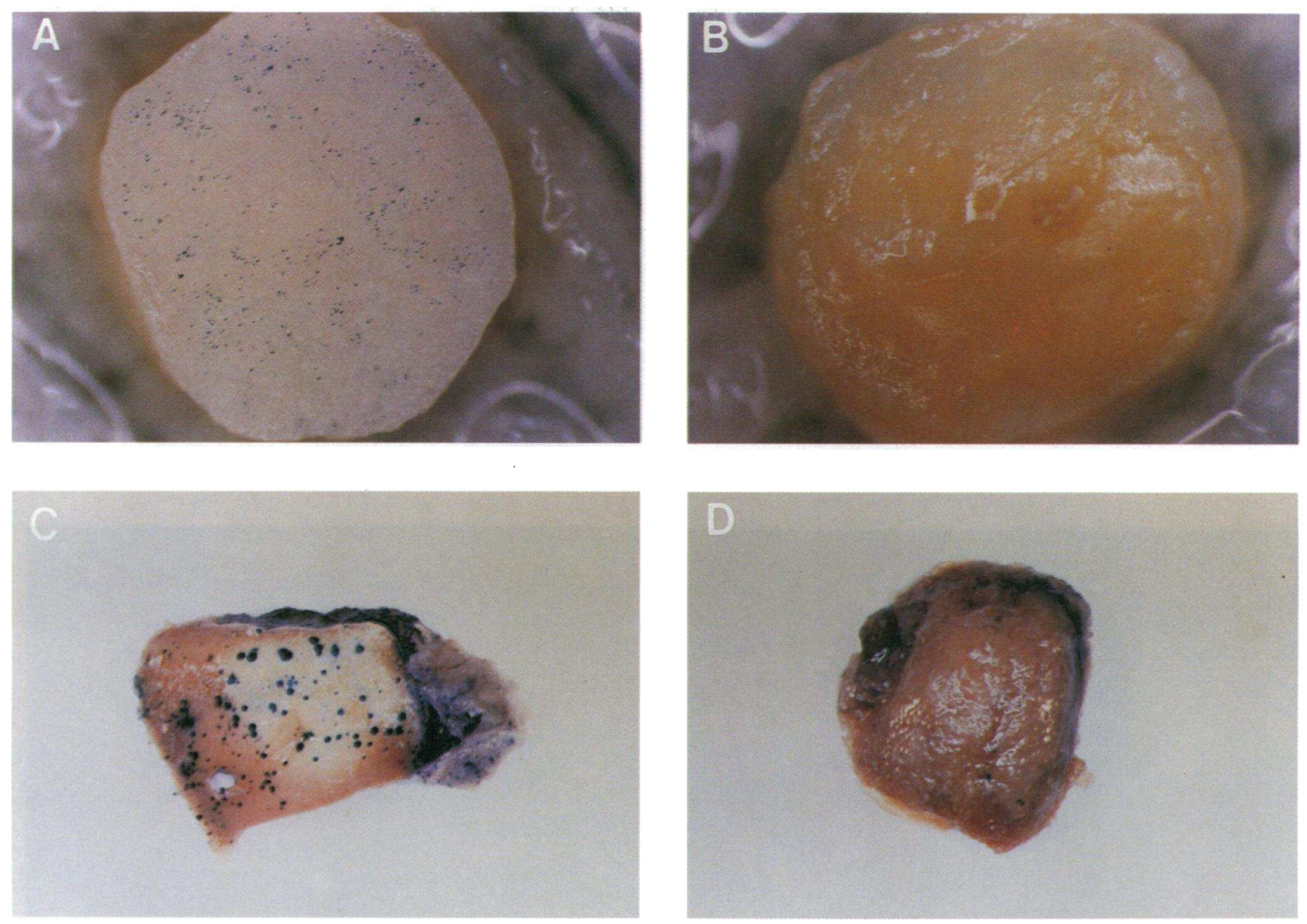

Figure 2. Expression of transgenic lacZ by human chondrocytes transplanted onto human cartilage organ cultures. Chondrocytes were transduced with Ad.RSV $n$ tlacZ at a an moi of $10^{3}$ and $24 \mathrm{~h}$ later were seeded onto the surface of cartilage organ cultures. Cultures were examined $8 \mathrm{~d}$ after transplantation by reaction with X-gal and photographed using stereoscopic microscopy (SMZ-U; Nikon Inc.). (A) Samples seeded with transduced cells exhibited multiple foci of cells with blue nuclei present across the articular surface of the cartilage, $\times 25$. $(B)$ Controls seeded with mockinfected chondrocytes exhibited no blue stain, $\times 25 .(C)$ Chondrocytes transduced with the vector Ad.CMV lacZ were also able to focally adhere to the articular surface and mediate expression of lacZ (cytoplasmic staining) in organ cultures with underlying subchondral bone left intact, $\times 10$. $(D)$ Controls had no evidence of $l a c Z$ positive cells along the articular surface, $\times 10$.

on the surface of OA human articular cartilage. $24 \mathrm{~h}$ after infection, chondrocytes were transplanted onto the surface of OA cartilage and maintained as sustained organ cultures for $8 \mathrm{~d}$. Examination of cartilage organ cultures for the presence of lacZtransduced cells $8 \mathrm{~d}$ after transplantation showed cells with blue nuclei distributed across the entire articular surface, indicating sustained lacZ expression during the observation period (Fig. 2 A). Cartilage samples seeded with mock-transfected cells showed no evidence of cells with blue staining nuclei (Fig. 2 $B$ ), indicating that $l a c Z$ expression on the articular surface was specific for the Ad.RSV ntlacZ-transduced chondrocytes. Examination of cartilage organ cultures containing intact subchondral bone also revealed multiple foci of blue staining chondrocytes (transduced with Ad.CMV lacZ) present on the articular surface (Fig. $2 \mathrm{C}$ ). Control samples seeded with mocktransfected cells were negative for blue-staining cells (Fig. 2 $D)$. These results support the concept that transduced chondrocytes can adhere to the surface of human OA cartilage, remain viable, and continue to express transgenic protein.

Scanning electron microscopy. Cartilage organ cultures seeded with lacZ-transduced chondrocytes (Fig. $3 A$ ) were then examined by scanning electron microscopy. Examination of the OA cartilage organ cultures revealed that foci of transduced chondrocytes were present across the articular surface of seeded disks (Fig. $3 B$ and $C$ ) but were not found on unseeded control disks (Fig. $3 D$ ). Fig. $3 B$ shows transplanted chondrocytes present on the surface of OA articular cartilage organ cultures that are embedded within a territorial extracellular matrix. At a higher magnification (Fig. $3 C$ ) adherent cells display an irregular surface as well as interconnecting cytoplasmic processes, characteristics of viable chondrocytes from OA human articular cartilage (27). A considerable amount of matrix material resembling collagen fibers also surrounds each cell. These results confirm the results obtained by stereoscopic microscopy and suggest that transduced chondrocytes can both adhere to and integrate into the surface of OA cartilage presumably through the elaboration of new ECM proteins.

Transduction of chondrocytes with the human IL-1 ra cDNA. The purpose of these experiments was to determine if chondrocytes transduced with the human IL1-ra cDNA and transplanted onto the surface of cartilage organ cultures can appropriately process and secrete a biologically active human cytokine antag- 

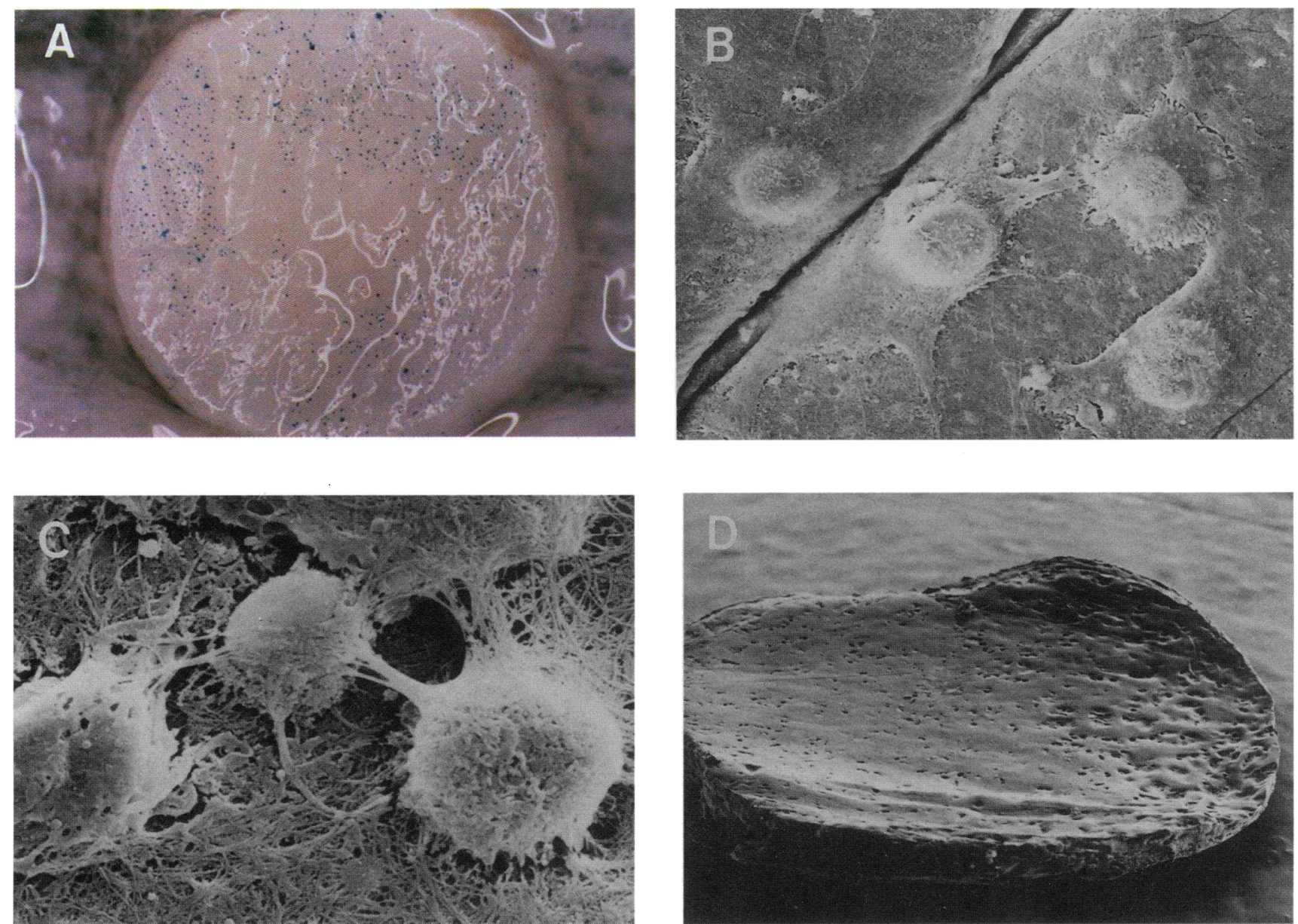

Figure 3. Analysis of cartilage organ cultures by scanning electron microscopy. (A) OA cartilage samples were seeded with lacZ-transduced chondrocytes and reacted with X-gal after $8 \mathrm{~d}$ of sustained organ culture, then processed for scanning electron microscopy as described in Methods. $\times 25$. $(B)$ Examination of transduced chondrocytes present on the surface of the cartilage organ cultures by scanning electron microscopy revealed that transplanted chondrocytes were adherent to the surface and embedded in an extracellular matrix. $\times 940$. (C) Scanning electron microscopy at higher magnification showed transduced chondrocytes with active cytoplasmic processes and evidence of mature collagen bundles surrounding the transplanted cells. $\times 3,790$. (D) Scanning electron microscopy of the entire surface of unseeded cartilage (identical to that shown in Fig. $1 B$ )

showed the surface of the tissue to be acellular. $\times 32$.

onist. Cartilage organ cultures containing chondrocytes transduced with Ad.RSVIL-1ra expressed and secreted on an average $1 \mu \mathrm{g} / \mathrm{ml}$ of human IL-1ra over a 10-d period; whereas cartilage cultures containing chondrocytes transduced with Ad.RSV $n$ tlac $Z$ produced on an average $100 \mathrm{pg} / \mathrm{ml}$ of IL-1ra (Fig. 4, ANOVA: $P<0.0001, F=56.7, n=16$ ). Identical groups of organ cultures continuously exposed to recombinant human IL- $1 \alpha(500 \mathrm{U} / \mathrm{ml})$ showed no statistically significant increase in the expression of human IL-1ra (Student-Newman-Keuls method of pairwise multiple comparison: $P<0.05$ ). Thus, transduced chondrocytes growing on the articular surface of OA cartilage can appropriately express, process, and secrete an immunoreactive transgenic protein. Furthermore, the expression of the transgenic IL-1 ra is independent of endogenous regulation and can be sustained at high levels for at least 10 in vitro.

Chondrocytes transduced with the human IL-1 ra cDNA inhibit IL-1 effects in OA cartilage cultures. To examine whether transplantation of chondrocytes transduced with the IL-1ra cDNA is chondroprotective, cartilage cultures were incubated in the presence or absence of exogenous $\mathrm{IL}-1 \alpha(500 \mathrm{U} / \mathrm{ml})$ for
$10 \mathrm{~d}$ and total proteoglycan degradation of tissue was determined. As shown in Fig. 5, OA organ cultures seeded with IL1ra-transduced chondrocytes and treated with exogenous IL-1 lost a mean of $11.7 \%$ of total proteoglycan content over the 10 d period of observation. Identical OA organ cultures seeded with lacZ-transduced chondrocytes lost a mean of $23.3 \%$ total proteoglycan content over the same time period. Control cultures containing lacZ- or IL-1ra-transduced chondrocytes that were unstimulated with IL-1 had a mean total proteoglycan loss of $11.2 \%$. These data indicate that chondrocytes transduced with the IL-1 ra cDNA when present as integrated cells on the surface of OA articular cartilage were able to inhibit IL-1-mediated chondrocyte degradation of ECM at a statistically significant level (ANOVA: $P<0.0001, F=12.5, n=34$; Student-Newman-Keuls method of pairwise multiple comparisons: $P$ $<0.05$ ). The results obtained from the unstimulated control cultures indicate that the increased loss of ECM from the IL1-stimulated cultures was specific for the exogenous cytokine. These experiments indicate that transduced chondrocytes are able to express, process, and secrete a biologically active 


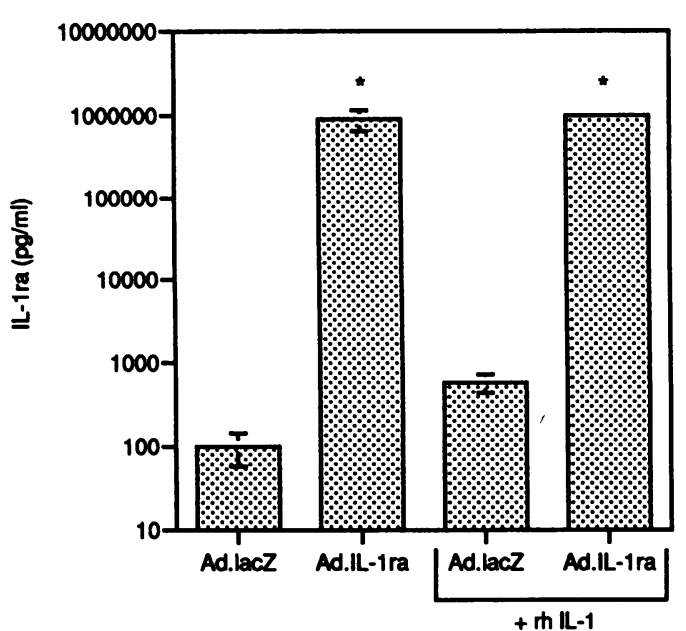

Figure 4. Expression of human IL-1 ra by transduced chondrocytes transplanted onto cartilage organ cultures. Approximately $10^{6}$ transduced chondrocytes were applied to the surface of each cartilage organ culture. $24 \mathrm{~h}$ after the cells had become adherent to the surface of the cartilage the organ cultures were maintained in complete medium in the presence or absence of recombinant human IL-1 $\alpha(50 \mathrm{U} / \mathrm{ml}$; Genzyme Corp. Cambridge, MA) for a period of $10 \mathrm{~d}$. The conditioned medium was then recovered from the organ cultures and the amount of soluble IL1ra was determined by ELISA (R\&D Systems Inc.). Results are expressed as picograms IL-1ra per ml. Tissues treated with Ad.RSVILlra-transduced cells produced significantly more IL-1ra than Ad.RSV $n$ tlac $Z$ controls with or without stimulation with recombinant human IL-1 (rhIL-1) (ANOVA: $F=56.7, n=16, P<0.0001$; StudentNewman-Keuls method of pairwise multiple comparisons: $P<0.05$ ). Mean values $( \pm \mathrm{SE})$ not statistically different are indicated by asterisks.

transgenic protein, and this protein has biological effects in the context of the microenvironment present across the surface of OA cartilage organ cultures.

\section{Discussion}

Strategies aimed at the development of chondroprotective biological agents for the treatment of human OA face the challenge of delivering these agents directly to the surface of abnormal articular cartilage. Previous work has indicated that chondrocytes participate directly in the pathophysiological events that trigger ECM destruction in hyaline cartilage (1-6). Current methods (systemic or intraarticular administration) for the introduction of biological agents into the joint space may fail to provide sustained proximal effects to the ECM within the microenvironment surrounding articular chondrocytes, presumably the area of greatest risk for the destructive effects mediated directly by the OA chondrocytes. This report describes a method of ex vivo gene transfer to articular chondrocytes that may allow circumvention of many of the problems inherent in the delivery of recombinant proteins to articular cartilage.

In this report we have modeled in vitro a method for the transduction and transplantation of genetically modified chondrocytes onto the surface of OA articular cartilage using a continuous cartilage organ culture system. As determined by in situ staining for $l a c Z$ protein, the transduced chondrocytes adhered to $O A$ cartilage, remained viable, and expressed transgenic protein for at least $8 \mathrm{~d}$ of continuous culture (Fig. $2 \mathrm{~A}$ ). Examination of the seeded cartilage tissues by scanning electron micros-

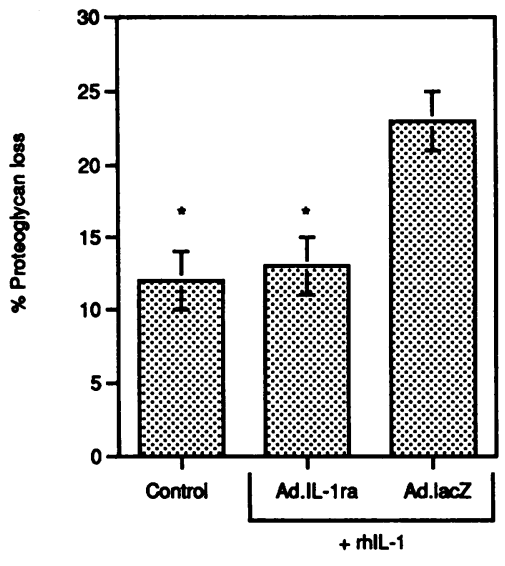

Figure 5. Transplantation of transduced chondrocytes overexpressing human IL-1ra protects cartilage organ cultures from IL-1-mediated proteoglycan degradation. Cartilage organ cultures seeded with $10^{6}$ Ad.RSVIL-1ra or Ad.RSV $n$ tlacZ-transduced chondrocytes were maintained in complete medium in the presence or absence of recombinant human IL-1a ( $50 \mathrm{U} /$ ml, Genzyme Inc.) for a period of $10 \mathrm{~d}$. The media and cartilage organ tissue were recovered and analyzed separately for total proteoglycan content using a 1,9dimethylmethylene blue dye binding assay (described in Methods). The percentage of proteoglycan loss in the organ culture system was calculated as the ratio of total proteoglycan in the conditioned media versus the total amount of proteoglycan present in the tissue + media. Tissues treated with Ad.RSVIL-1 ra-transduced cells had significantly less proteoglycan loss than Ad.lacZ-treated controls (ANOVA: $F=12.5, n$ $=34, P<0.0001$; Student-Newman-Keuls method of pairwise multiple comparisons: $P<0.05$ ). No significant differences were observed between lacZ- and IL-1ra-transduced controls, and these values were pooled for purposes of statistical analyses. The data shown are means \pm SE, and mean values not statistically different are indicated by asterisks. rhIL-1, recombinant human IL-1.

copy provided additional evidence that the transplanted cells maintained viability and were able to begin the process of integration into the existing surface matrix through the elaboration of new ECM.

We chose to examine the effects of adenoviral-mediated overexpression of the human IL-1 ra as a prototypic chondroprotective protein. The recombinant IL-1ra protein is under study as a potentially therapeutic biological agent able to counteract in vivo the proinflammatory effects of IL-1, a cytokine recognized to function as a central mediator of inflammation and cartilage degradation (14-19). Human chondrocytes transduced with an adenovirus containing the cDNA for IL-1 ra (Ad.RSV hIL-1 ra) were able to elaborate large amounts of this potentially chondroprotective transgenic protein and continued to secrete this protein after adherence to the cartilage surface when maintained as a continuous cartilage organ culture. The results of these studies indicate that transduced chondrocytes express quantities of biologically active IL-1ra that is sufficient to counteract the IL-1 induced proteoglycan degradation from OA cartilage organ cultures over a period of at least $10 \mathrm{~d}$ of continuous exposure to the proinflammatory signal. This is a noteworthy achievement in that it has not been possible to demonstrate chondroprotection by delivery of recombinant IL-1ra using traditional routes of delivery $(28,29)$.

Transplantation of heterologous or autologous chondrocytes has already been evaluated as a treatment for the repair of focal articular defects in several animal models (30-32). Recently, autologous chondrocyte transplantation has been successfully applied to the treatment of isolated cartilage defects in a series of human patients (13). Because cartilage degradation in OA is the end result of a complex interaction of catabolic and anabolic 
processes, it remains to be seen whether the transplantation of autologous chondrocytes alone would be sufficient to allow appropriate repair of OA cartilage. It is reasonable to hypothesize that it will be necessary to initially inhibit the degradative process before appropriate repair can occur as a consequence of cellular transplantation. In this study we chose to combine chondrocyte transplantation with ex vivo gene transfer as a means to augment the biological effects of these cells, specifically in terms of the ability of transplanted cells to provide a sustained source of chondroprotective proteins.

In general, ex vivo gene transfer strategies have used recombinant retroviruses as gene transfer vectors $(8-11)$. Our results using recombinant adenoviruses as ex vivo gene transfer vectors for chondrocytes suggest several potential advantages over the use of recombinant retroviruses. Primary among these is the high efficiency of transduction ( $>90 \%$ at an moi of $10^{3}$ ) that can be achieved without the need for prolonged monolayer culture or the use of selectable marker genes. Short term monolayer culture allows the chondrocytes to retain many of the markers associated with the differentiated chondrocyte phenotype and in turn this may facilitate adherence and integration of the transduced cells onto the articular surface at the time of transplantation. Other advantages include virtually no risk of insertional mutagenesis and the possibility of coinfection using multiple vectors to mediate the simultaneous expression of two or more transgenic proteins. Our findings indicate that ex vivo transduction followed by autologous transplantation of human chondrocytes is a viable strategy for intraarticular gene therapy. Further development of this system using in vivo animal models may allow the development of novel molecular approaches for the treatment of OA and a variety of other human arthropathies.

\section{Acknowledgments}

The authors thank Dr. John Henke (St. Joseph Mercy Hospital, Ypsilanti, MI) for critical discussions and assistance in acquisition of tissue samples, and J.-M. Quevedo for excellent secretarial support.

This study was supported in part by The National Institutes of Health (R55 AR42412) and the University of Michigan Multipurpose Arthritis and Musculoskeletal Diseases Center (P60 AR20557).

\section{References}

1. Steinberg, J. J., and C. B. Sledge. 1991. Chondrocyte mediated cartilage degradation: regulation by prostaglandin $\mathrm{E}_{2}$, cyclic AMP and interferon $\alpha . J$. Rheumatol. 18(Suppl. 27):63-65.

2. Dodge, G. R., and A. R. Poole. 1989. Immunohistochemical detection and immunochemical analysis of type II collagen degradation in human normal, rheumatoid, and osteoarthritic articular cartilages and in explants of bovine articular cartilage culture with interleukin 1. J. Clin. Invest. 83:647-661.

3. Fell, H. B., and R. W. Jubb. 1977. The effect of synovial tissue on the breakdown of articular cartilage in organ culture. Arthritis Rheum. 20:1359-1371.

4. Hamerman, D. 1969. Cartilage changes in the rheumatoid joint. Clin. Orthop. Relat. Res. 64:91-97.

5. Bora, F. W., Jr., and G. Miller. 1987. Joint physiology, cartilage metabolism, and the etiology of osteoarthritis. Hand Clinics. 3:325-336.

6. Bland, J. H., and S. M. Cooper. 1984. Osteoarthritis: a review of the cell biology involved and evidence for reversibility. Management rationally related to known genesis and pathophysiology. Semin. Arthritis Rheum. 14:106-132.

7. Bandara, G., P. D. Robbins, H. I. Georgescu, G. M. Mueller, J. C. Glorioso, and C. H. Evans. 1992. Gene transfer to synoviocytes: prospects for gene treatment of arthritis. DNA Cell Biol. 11:227-231.
8. Bandara, G., G. M. Mueller, J. Galea-Lauri, M. H. Tindal, H. I. Georgescu, M. K. Sucharek, G. L. Hung, J. C. Glorioso, P. D. Robbins, and C. H. Evans. 1993. Intraarticular expression of the interleukin-1 receptor antagonist protein by ex vivo gene transfer. Proc. Natl. Acad. Sci. USA. 90:10764-10768.

9. Roessler, B. J., E. D. Allen, J. M. Wilson, J. W. Hartman, and B. L. Davidson. 1993. Adenoviral-mediated gene transfer to rabbit synovium in vivo. J. Clin. Invest. 92:1085-1092.

10. Roessler, B. J., J. W. Hartman, D. K. Vallance, J. M. Latta, S. L. Janich, and B. L. Davidson. 1995. Inhibition of interleukin-1 induced effects in synoviocytes transduced with the human IL-1 receptor antagonist cDNA using an adenoviral vector. Hum. Gene Ther. 6:307-316.

11. Hung, G. L., J. Galea-Lauri, G. M. Mueller, H. I. Georgescu, L. A. Larkin, M. K. Sucharek, M. H. Tindal, P. D. Robbins, and C. H. Evans. 1994. Suppression of intra-articular responses to interleukin-1 by transfer of the interleukin- 1 receptor antagonist gene to synovium. Gene Ther. 1:64-69.

12. Evans, C. H., and P. D. Robbins. 1994. Gene therapy for arthritis. In Gene Therapeutics: Methods and Applications of Direct Gene Transfer. J. A. Wolff, editor. Birkhauser Boston, Inc., Cambridge, MA. 320-343.

13. Brittberg, M., A. Lindahl, A. Nilsson, C. Ohlsson, O. Isaksson, and L. Peterson. 1994. Treatment of deep cartilage defects in the knee with autologous chondrocyte transplantation. N. Engl. J. Med. 331:889-895.

14. Dinarello, C. A. 1989. Interleukin-1 and its biologically related cytokines. Adv. Immunol. 44:153-205.

15. Arend, W. P., and J.-M. Dayer. 1990. Cytokines and cytokine inhibitors or antagonists in rheumatoid arthritis. Arthritis Rheum. 33:305-315.

16. Smith, R. J., N. A. Rohloff, L. M. Sam, J. M. Justen, M. R. Diebel, and J. C. Cornette. 1989. Recombinant human interleukin- $\alpha$ and recombinant human interleukin- $1 \beta$ stimulate cartilage matrix degradation and inhibit glycosaminoglycan synthesis. Inflammation. 13:367-382.

17. Kumkumian, G. K., R. Lafyatis, E. F. Remmers, J. P. Case, S. J. Kim and R. L. Wilder. 1989. Platelet-derived growth factor and IL-1 interactions in rheumatoid arthritis. Regulation of synoviocyte proliferation, prostaglandin production, and collagenase transcription. J. Immunol. 143:833-837.

18. Gilman, S. C. 1987. Activation of rabbit articular chondrocytes by recombinant human cytokines. J. Rheumatol. 14:1002-1007.

19. Dayer, J. M., B. de Rochemonteix, B. Burrus, S. Demczuk, and C. A Dinarello. 1986. Human recombinant interleukin 1 stimulates collagenase and prostaglandin $\mathrm{E}_{2}$ production by human synovial cells. J. Clin. Invest. 77:645648

20. Dinarello, C. A., and R. C. Thompson. 1991. Blocking IL1: interleukin1 receptor antagonist in vivo and in vitro. Immunol. Today. 12:404-410.

21. Schwab, J. H., S. K. Anderle, R. R. Brown, F. G. Dalldorf, and R. C Thompson. 1991. Pro- and anti-inflammatory roles of interleukin-1 in recurrence of bacterial cell wall induced arthritis in rats. Infect. Immunol. 59:4436-4442.

22. Dripps, D. J., B. J. Brandhuber, R. C. Thompson, and S. P. Eisenberg. 1991. Interleukin-1 (IL-1) receptor antagonist binds to the $80 \mathrm{kDa}$ IL-1 receptor but does not initiate IL-1 signal transduction. J. Biol. Chem. 266:10331-10336. 23. Arend, W. P. 1993. Interleukin-1 receptor antagonists. Adv. Immunol. 54:167-227.

24. Hurwitz, D. R., and G. Chinnadurai. 1985. Evidence that a second tumor antigen coded by adenovirus early gene region E1a is required for efficient cell transformation. Proc. Natl. Acad. Sci. USA. 82:163-167.

25. Walsh, C., and C. L. Cepko. 1988. Clonally related cortical cells show several migrations patterns. Science (Wash. DC). 241:1342-1345.

26. Baragi, V. M., H. Jordan, and R. R. Renkiewicz. 1992. A protocol for rapid screening of proteoglycan-degrading metalloproteinase inhibitors. J. Pharmacol Methods. 27(2):1-5.

27. Weiss, C., and S. Mirow. 1972. An ultrastructural study of osteoarthritic changes in the articular cartilage of human knees. J. Bone J. Surg. Br. Vol. 54:954-972a (Abstr.)

28. Wooley, P. H., J. D. Whalen, D. L. Chapman, A. E. Berger, K. A. Richard, D. G. Aspar, and N. D. Staite. 1993. The effect of an interleukin-1 receptor antagonist protein on type II collagen-induced arthritis and antigen-induced arthritis in mice. Arthritis Rheum. 36:1305-1314.

29. Lewthwaite, J. C., S. M. Blake, T. E., Hardingham, and B. Henderson. 1993. The effect of interleukin-1 receptor antagonist on the progression of antigeninduced arthritis in the rabbit. Trans. Orthop. Res. Soc. 18:237.

30. Chesterman, P. J., and A. U. Smith. 1968. Homotransplantation of articular cartilage and isolated chondrocytes: an experimental study in rabbits. J. Bone J. Surg. Br. Vol. 50:184-97.

31. Green, W. T., Jr. 1977. Articular cartilage repair: behavior of rabbit chondrocytes during tissue culture and subsequent allografting. Clin. Orthop. 124:237-250.

32. Aston, J. E., and G. Bentley. 1986. Repair of articular surfaces by allografts of articular and growth-plate cartilage. J. Bone J. Surg. Br. Vol. 68:29-35. 